\title{
The Effects of Situational and Individual Factors on Algorithm Acceptance in COVID-19-Related Decision-Making: A Preregistered Online Experiment
}

\author{
Sonja Utz ${ }^{1,2}$, Lara N. Wolfers ${ }^{2}{ }^{(}$, and Anja S. Göritz ${ }^{3(}$ \\ 1 Leibniz-Institut für Wissensmedien, Schleichstr. 6, 72076 Tübingen, Germany \\ 2 University of Tübingen, 72074 Tübingen, Germany \\ 3 University of Freiburg, Occupational and Consumer Psychology, 79106 Freiburg, Germany
}

\section{Abstract}

In times of the COVID-19 pandemic, difficult decisions such as the distribution of ventilators must be made. For many of these decisions, humans could team up with algorithms; however, people often prefer human decision-makers. We examined the role of situational (morality of the scenario; perspective) and individual factors (need for leadership; conventionalism) for algorithm preference in a preregistered online experiment with German adults ( $n=1,127)$. As expected, algorithm preference was lowest in the most moral-laden scenario. The effect of perspective (i.e., decision-makers vs. decision targets) was only significant in the most moral scenario. Need for leadership predicted a stronger algorithm preference, whereas conventionalism was related to weaker algorithm preference. Exploratory analyses revealed that attitudes and knowledge also mattered, stressing the importance of individual factors.

Keywords: algorithm preference, decision-making, perspective, need for leadership, conventionalism

In December 2019, a virus causing the coronavirus disease 2019 (COVID-19) first appeared in Wuhan, China. It rapidly spread around the world, and the outbreak was declared a pandemic by the World Health Organization on March 11, 2020 (WHO, 2020). By April 2021, more than 2.9 million people had died from COVID-19 ("COVID-19 pandemic," 2021).

CONTACT Sonja Utz (D) • Leibniz-Institut für Wissensmedien • Schleichstr. 6 • 72076 Tübingen, Germany • s.utz@iwm-tuebingen.de 
During the pandemic, many decisions with far-reaching consequences had to be made: Governments had to decide on which restrictions to impose on the population (e.g., closing schools, imposing curfews) and when to loosen them (Gollwitzer et al., 2020); physicians had to decide who gets a potentially life-saving ventilator in overwhelmed hospitals, and civil servants at ministries had to decide which businesses receive financial support after being forced to close for months.

Algorithms could have helped in making these and related decisions. Algorithms process "(big) data captured through digitized devices" and use past behavior to predict future events (Newell \& Marabelli, 2015, p. 4). Algorithms might be especially helpful in situations like the pandemic in which human deciders lack experience. However, prior research has shown that people are often algorithm averse (Castelo et al., 2019; Dietvorst et al., 2015). For policymakers, it is thus important to know under which circumstances algorithmic decision-making and, more specifically, different combinations of human-algorithm teaming would be accepted. Two groups are important in this context: (1) the decision-makers who could now (also) rely on algorithmic input and (2) the decision targets whose outcomes depend on the decisions made. The question of human-machine teaming has been addressed in the field of human-machine communication, but usually from the perspective of the decision-maker and with a focus on more agentic and anthropomorphic virtual teammates such as robots (Calhoun et al., 2019; Matthews et al., 2020). Work on algorithm acceptance has addressed both perspectives, albeit usually not in the same study; we thus build on work on algorithm acceptance to identify relevant situational and individual influence factors and develop a new measure that goes beyond a dichotomous human versus algorithm choice, which enables assessment of the preference for different human-algorithm teaming combinations. This approach promotes ecological validity because algorithms rarely make decisions on their own but usually function as advisors or recommenders.

Specifically, we examined morality of the decision scenario and the perspective as situational factors and both conventionalism and need for leadership as individual factors. Previous work has found that people are more averse to algorithms when moral decisions must be made (Bigman \& Gray, 2018; Castelo et al., 2019). We aim to extend this work by examining whether it matters from which perspective the algorithmic decision-making is judged: Do people show a higher preference for algorithms if they take the decision-maker's perspective compared to the perspective of the target of the decision? A physician, for example, might perceive the algorithm as an "advanced tool" (Matthews et al., 2020, p. 234), whereas patients might perceive it as a threat and prefer a human deciding upon their life.

Most prior work on algorithm aversion looked at characteristics of the algorithm or the human counterpart, but less on characteristics of the individuals choosing between algorithmic and human decision-making (Jussupow et al., 2020). In this paper, we look at conventionalism - an individual characteristic that should favor human decision-making - and an individual's need for leadership. Need for leadership might be especially relevant in times of COVID-19 because this need is stronger in times of crisis (Mulder \& Stemerding, 1963).

\section{Algorithm Aversion and Appreciation}

The term algorithm aversion has been coined by Dietvorst et al. (2015) to describe the sometimes not rational reaction of users toward algorithms, such as a preference for human 
decision-making even if the algorithmic decisions are superior to the ones made by humans. Research on algorithm aversion has yielded inconsistent results, documenting also cases in which individuals prefer algorithms to humans (algorithm appreciation, e.g., Dietvorst et al., 2016; Logg et al., 2019).

Jussupow et al. (2020) reviewed experimental work in this field to identify characteristics of the algorithm versus a human decision-maker that predict whether algorithm aversion or appreciation occurs. Frequently studied characteristics of the algorithms are their agency or performance, and frequently studied characteristics of the human decision-maker are their involvement in the development or training of the algorithm or their expertise. Algorithmic agency led to aversion; thereby people were especially averse to algorithms making decisions independently (Jussupow et al., 2020). Moreover, algorithm aversion was lower when people perceived the algorithms as performing well and possessing human capabilities, whereas higher expertise of the human agent increased algorithm aversion. Human involvement only had an indirect effect via algorithm agency and capabilities (Jussupow et al., 2020).

\section{Human-Algorithm-Teaming}

Algorithms rarely make decisions on their own; the topic of various forms and degrees of human-algorithm teaming has therefore received attention. Starke and Lünich (2020) showed that pure algorithmic decisions of the European Union would be perceived as illegitimate, whereas hybrid decisions are perceived as equally legitimate as human-only decisions. The authors did, however, not specify the nature of the human-algorithm teaming. There is some conceptual work on this topic. Madni and Madni (2018) provide a framework that distinguishes the roles of humans and machines; frequent roles are the human as supervisor and the machine either in an active or passive monitoring role. Van der Waa et al. (2020) focus on moral decisions and distinguish between human moral decisionmaking, supported moral decision-making, co-active moral decision-making, and autonomous moral decision-making, in which the artificial moral agent makes moral decisions on its own. These conceptual papers give examples of the different configurations and discuss the advantages and disadvantages of the different patterns, but they do not examine which factors determine the preference for lower or higher algorithmic input. In the paper at hand, we fill this gap and use the COVID-19 pandemic as a setting to explore the role of situational and individual factors.

\section{Situational Factors}

\section{Morality/Severity of the Decision}

The first factor we considered was the moral dimension of the decision task. According to Schein and Gray (2018), the judgment of whether a situation is morally laden depends on whether there is harm involved and how severe the consequences are. Although there are representative surveys showing that people are less likely to accept algorithmic decision-making in situations with severe consequences for humans such as parole, medical diagnoses and decisions, or personal finance scores (Grzymek \& Puntschuh, 2019; Longoni et al., 2019; Smith, 2018), there is surprisingly little experimental research on this 
topic. Bigman and Gray (2018) concluded that people display algorithm aversion when the algorithms make moral decisions such as deciding over parole, or medical decisions. This conclusion might imply a comparison with less moral-laden decisions; however, the nine studies reported in the paper all used moral decision-making situations. In a similar vein, Longoni et al. used only scenarios involving medical decision-making.

In contrast, in one of the few papers that showed high algorithm acceptance across several studies most decisions were objective judgment tasks (such as the weight of a person) or had mild consequences (i.e., suggesting a dating partner; Logg et al., 2019). Castelo et al. (2019) compared subjective and objective decision-making tasks and found that people preferred algorithms for objective tasks. These objective tasks are less moral-laden. Taken together, prior findings warrant the expectation that people are less algorithm averse if decisions involve less morality. This was, however, not directly tested in any of the studies.

Work on human-machine teaming has, to our knowledge, not systematically compared scenarios varying in morality, but it assumes that it will take some time until artificial moral agents reach human or even super-human levels of moral decision-making; consequentially, human-machine teaming is needed (van der Waa et al., 2020). This work, thus, also implicitly assumes that people prefer less algorithmic involvement in moral decisions.

To systematically explore the role of morality, the work at hand varies the severity and thus morality of the decision's consequences across three scenarios: (1) a scenario about the distribution of ventilators among patients, (2) a scenario about financial support for businesses suffering economically from the COVID-19 pandemic, and (3) a scenario on curfew rules for members of risk groups. Since the ventilator scenario is about life and death, it was considered the most morality-laden scenario with the most severe consequences and should thus be met with the lowest preference for algorithms. We had no prediction about the order of the other two scenarios.

H1: Preference for algorithmic decision-making is lowest in the ventilator scenario (highest morality and severity).

\section{Perspective}

We assumed that it matters whether a person is the target of a decision or the decisionmaker. An alternative explanation for the algorithm appreciation found in the studies reported by Logg et al. (2019) is that the authors used the advice-taking paradigm; participants had to make decisions and received advice stemming allegedly from an algorithm versus a human. In such a scenario, the algorithm might help the human make better decisions. Decision-makers might thus activate an "advanced tool" or even a "teammate" mental model (Matthews et al., 2020, p. 234) and be willing to share the responsibility for a decision with an algorithm. Targets of a decision, by contrast, might view the algorithm as a threat and be more interested in self-benefit than in the most accurate or efficiently made decision. Self-serving biases have been shown in the domain of preference of fairness rules (Messick \& Sentis, 1983) and have been reported as "outcome favorability bias" in studies on algorithm acceptance (Wang et al., 2020, p. 1). In the context of COVID-19, elderly people with several diseases might, for example, be afraid that the "objectively" best decision is to give the ventilator to a younger person with a higher chance of surviving a COVID-19 infection. In their mental model, the algorithm might form a threat to their life. They might, 
however, hope to be able to influence a human decision-maker, for example, by appealing to empathy or by bribery. Therefore, we expect a higher preference for algorithms among decision-makers relative to targets of decisions.

H2: Preference for algorithmic decision-making is higher in the decision-maker (versus target of decision) condition.

Next to these situational factors, there might be inter-individual differences that predict the preference for algorithmic versus human decision-making.

\section{Individual Factors}

\section{Conventionalism}

Algorithms and artificial intelligence are quite new phenomena. To a layperson, it is often not clear how algorithms make complex decisions (Grzymek \& Puntschuh, 2019). GrgicHlaca et al. (2018) found that conservatism affected the perceived fairness of algorithms. Similarly, we expected that people scoring high on conventionalism, that is, people who prefer to stick to established procedures and norms, are more averse toward these new and non-transparent technologies and hence show a weaker preference for algorithmic decision-making.

H3: People higher in conventionalism show a weaker preference for algorithmic decision-making.

\section{Need for Leadership}

A personality factor that might be relevant in times of crisis is the need for leadership. Crises are characterized by uncertainties; this holds especially for the novel coronavirus. At the time this study was conducted, nobody knew when a treatment or a vaccine would be found and how large the impact of the lockdowns on the economy or mental health of people would be. In times of crisis, people show a stronger need for leadership (Mulder \& Stemerding, 1963). People expect leaders to make clear and consistent decisions, as could be seen in the higher approval ratings of politicians who implemented tough measures in response to COVID-19 (Erlanger, 2020). Based on this assumption, one should expect that a higher need for leadership is related to a preference for human decision-making. However, it might also be the case that a higher need for leadership is related to a preference for algorithmic decision-making because algorithms might be perceived as more objective (in the sense of following the same rules all the time) and thus as giving clearer and more consistent guidance. It is also possible that need for leadership is related to higher endorsement for decisions made by one entity alone (versus a team), no matter whether it is a human or an algorithm. Since there are several possibilities, an open research question was posed:

RQ: Is need for leadership related to algorithm preference?

\section{Attitudes Toward Algorithms and Knowledge}

By way of exploration, the participants' attitudes toward algorithms and knowledge about algorithms were assessed. Concerning the attitudes, we covered the perceived decision 
quality (ability of algorithms to process large amounts of data/a wider variety of data types), different aspects of fairness, and perceived manipulability to explore whether the effects of the other variables remain robust when controlling for attitudes and knowledge. The focus of the experiment is - as can also be seen in the preregistration-however, on the situational and individual factors.

The hypotheses and research questions, operationalization, design, and analysis plan have been preregistered at https://aspredicted.org/zv2m2.pdf. ${ }^{1}$

\section{Method}

\section{Participants}

The study was approved by the local ethics committee of Leibniz-Institut für Wissensmedien (Knowledge Media Research Center), Tübingen. All 14,283 members of the WiSoPanel, an online access panel for non-commercial research (Göritz et al., 2021), were invited via email to participate. The experiment had a 3 (scenario: ventilator, financial support, curfew) $\times 2$ (perspective: target of the decision versus decision-maker) design. The scenario factor was a within-subjects factor; the perspective factor was manipulated between subjects. Only people who permitted the use of their data at the end of the questionnaire $(1,192$ women, 968 men) were retained. Most of the participants were German (96.6\%), 1.9\% were Austrian, $1 \%$ Swiss, and $0.4 \%$ from other countries. The largest group of participants $(31.7 \%)$ had a university degree, $23.8 \%$ A-Levels, $29.5 \%$ O-Levels, $11.4 \%$ had finished 9 years of school, $0.6 \%$ had no degree (yet), and $3.1 \%$ had a doctorate. The majority $(61.1 \%)$ were working, $20.4 \%$ were retired, $8.1 \%$ pupils/students, $5.7 \%$ unemployed, and $1 \%$ on parental leave.

\section{Procedure}

After reading the introduction and providing informed consent, respondents stated their preferences for algorithmic versus human decision-making in three COVID19-related decision scenarios. Depending on the experimental condition, participants either took the perspective of the target of the decision or of the decision-maker in all three scenarios.

\section{Independent Variables}

\section{Scenarios}

In the first scenario, participants were asked to imagine that there were more COVID-19 patients than ventilators, hence a decision about who gets a ventilator had to be made. In the second scenario, a decision about whether financial support should be granted to business owners who suffered financially from the COVID-19-induced closure of their business had to be made. In the last scenario, participants were told that the local public health departments would decide for individual members of risk groups whether they had to adhere to stronger curfew rules. 


\section{Perspective}

In the target of the decision condition, participants were asked to imagine that they were a COVID-19 patient, a business owner, or at higher risk to die from COVID-19, respectively for the three scenarios. In the decision-maker condition, participants were asked to imagine that they were a physician, a clerk, or an employee of the public health department, respectively.

\section{Measures}

\section{Algorithm Preference}

Since hitherto used measures are not equally applicable to decision-makers and decision targets and because we aimed to go beyond a dichotomous choice between human versus algorithm, we offered six combinations of human-algorithm teaming (see Table 1, top row) and gave people fine-grained options to indicate their preferences. Participants were instructed to distribute 100 points across the decision-making options. They could either assign 100 points to their favorite option or split the points across options. For the first three options, the algorithm made the final decision; for the last three options, a human made the final decision. In the two most extreme cases, the decision was made by the algorithm/ human alone. In the next option, in difficult cases, a human/an algorithm made a suggestion that could be incorporated by the algorithm/human; in the two options in the middle, the algorithm/human considered the suggestion of the human/algorithm in all decisions.

\begin{tabular}{|c|c|c|c|c|c|c|c|}
\hline \multirow[t]{2}{*}{ TABLE 1} & \multicolumn{7}{|c|}{$\begin{array}{l}\text { Mean Number of Points Given to the Six Decision Options Across Scenarios } \\
\text { and Perspective (Subsample With Correct Manipulation Checks) }\end{array}$} \\
\hline & & $\begin{array}{l}\text { Human } \\
\text { alone } \\
\text { (1) }\end{array}$ & $\begin{array}{c}\text { Human, } \\
\text { input from } \\
\text { algorithm } \\
\text { in difficult } \\
\text { cases } \\
\text { (2) }\end{array}$ & $\begin{array}{l}\text { Human but } \\
\text { always } \\
\text { input from } \\
\text { algorithm } \\
\text { (3) }\end{array}$ & $\begin{array}{l}\text { Algorithm } \\
\text { but always } \\
\text { input from } \\
\text { human } \\
\text { (4) }\end{array}$ & $\begin{array}{c}\text { Algorithm, } \\
\text { input from } \\
\text { human in } \\
\text { difficult } \\
\text { cases } \\
\text { (5) }\end{array}$ & $\begin{array}{c}\text { Algorithm } \\
\text { alone } \\
\text { (6) }\end{array}$ \\
\hline \multirow[t]{2}{*}{ Ventilator } & decision target & 21.42 & 25.75 & 28.44 & 12.07 & 6.37 & 5.96 \\
\hline & decision-maker & 12.57 & 24.21 & 34.20 & 13.08 & 7.42 & 8.53 \\
\hline Financial & decision target & 12.24 & 19.36 & 32.12 & 16.57 & 11.35 & 8.37 \\
\hline Support & decision-maker & 11.10 & 19.55 & 32.92 & 16.99 & 10.06 & 9.38 \\
\hline \multirow[t]{2}{*}{ Curfew } & decision target & 16.34 & 19.44 & 29.10 & 14.79 & 9.89 & 10.44 \\
\hline & decision-maker & 14.38 & 22.26 & 29.76 & 15.08 & 9.20 & 9.32 \\
\hline
\end{tabular}

Note: Sums not adding up exactly to 100 within some rows due to rounding.

\section{Need for Leadership}

We adapted five items from the interpersonal hierarchy expectation scale by Mast (2005) to the context of the COVID-19 pandemic (e.g., "If people work together on a task like the current corona crisis, it's best if one person is taking over the lead."). Respondents indicated their agreement with the statements on a scale ranging from $1=$ "strongly disagree" to $7=$ "strongly agree" ( $\alpha=.80, M=4.48, S D=1.26)$. 


\section{Conventionalism}

Conventionalism was measured with the 3-item scale by Beierlein et al. (2014). Agreement to statements like "It is always best to do things the usual way" was provided on a 7-point Likert scale $(\alpha=.83, M=3.65, S D=1.56)$.

\section{Attitude Toward Algorithms}

To measure attitude toward algorithms, 10 items were developed that covered various aspects of decision-making. Participants indicated whether they thought humans or algorithms would usually perform better on a certain aspect on a scale from 1 = "humans" to 3 = "humans and algorithms to the same degree" to 5 = "algorithms." A confirmatory factor analysis allowing for correlations between factors showed that, as expected, the 10 items loaded on five factors $\left(\chi^{2}[25, \mathrm{~N}=2147]=218.20, p<.001\right.$; CFI $=0.94$; RMSEA $\left.=.060\right)$, correctness of decision $(M=3.21, S D=0.75)$, procedural fairness (e.g., judge situations always along the same factors, $M=3.98, S D=0.92)$, multifactorial decision-making $(M=3.17, S D$ $=1.01)$, manipulability $(M=2.33, S D=1.00)$, and general fairness $(M=3.13, S D=0.87)$. For items and more results see https://osf.io/cx6z8/.

\section{Knowledge About Algorithms}

As a control variable, participants were asked to indicate their knowledge about algorithms (definition, methods, accuracy, fairness) on a scale from $1=$ very poor knowledge to $7=$ very good knowledge $(M=4.08, S D=1.53)$.

\section{Manipulation Checks}

Participants were asked whether they had been in the role of the patient or physician in Scenario 1, business owner or clerk in Scenario 2, a member of the group at higher risk of the coronavirus or employee at the public health department in Scenario 3. There was also a "don't know" option to reduce guessing. Participants were also asked to rank order the three scenarios in terms of severity and moral dimension by dragging and dropping them to the top, middle, or bottom position.

\section{COVID-19-Related Additional Measures}

Participants were asked for their subjective risk to get infected with COVID-19, whether they were at higher risk from coronavirus according to the criteria issued by the national Robert Koch institute (e.g., smoking, chronic medical condition), whether they had been infected with COVID-19, or whether close others had been infected or died. The likelihood of experiencing negative financial consequences due to the COVID-19 pandemic was assessed on a scale from 1 = "very unlikely" to 6 = "certainly." Of the final sample (see below), only 10 had been infected with COVID-19, 5.6\% reported one or more infected close others. Roughly a quarter considered it likely, very likely, or certain to become infected, and $44.7 \%$ were members of a risk group.

\section{Demographics}

The following demographics were retrieved from the panel data: year of birth, gender, country, education, and employment status. The intercorrelations between the central measures are displayed in Table 2. 


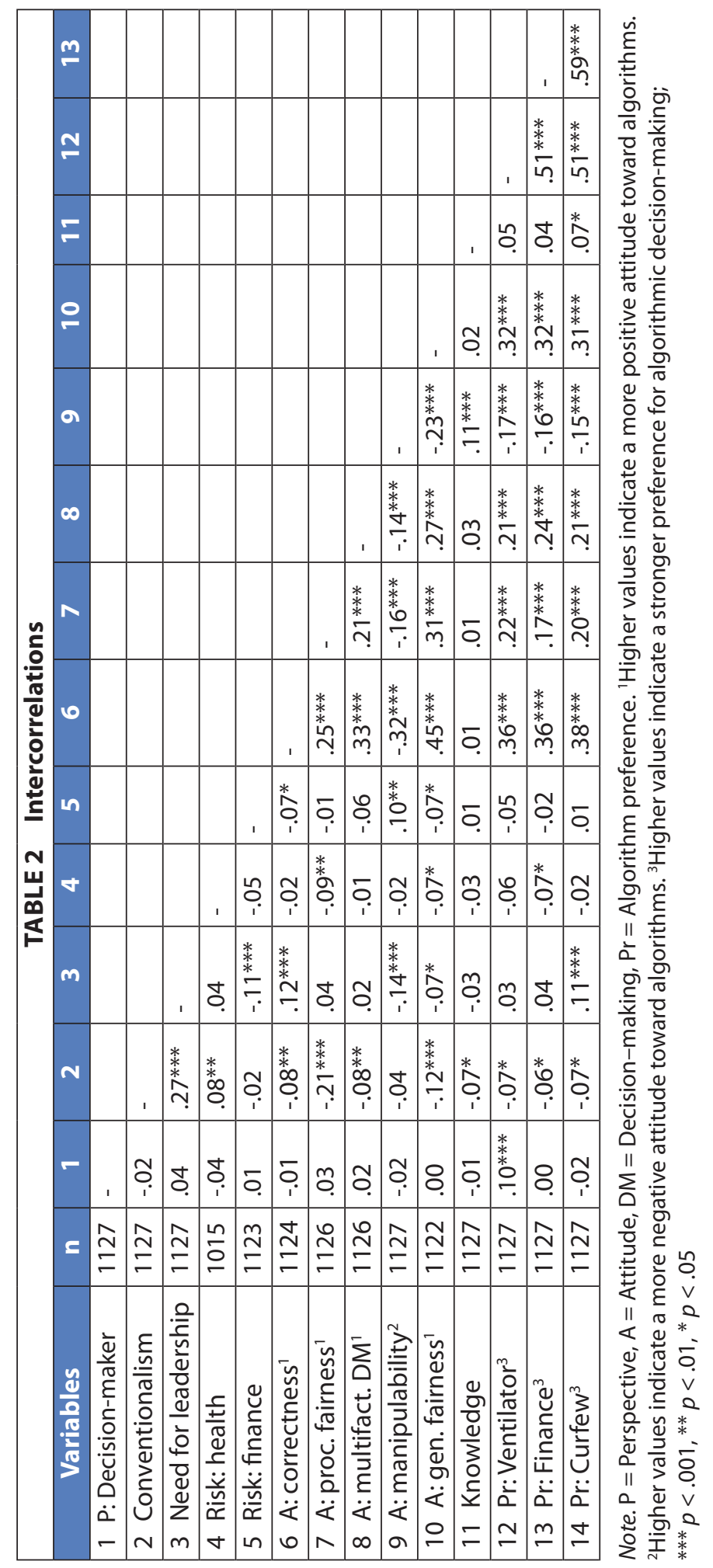




\section{Data Preparation}

Missings. Two participants were excluded because they had more than $30 \%$ missing values.

Construction of the dependent measure. As preregistered, we first inspected the distributions. To assess nuanced preferences, participants had the option to split 100 points across six options rather than choosing only one option. The majority (56 to 60\%, depending on the scenario) did not split but gave $100 \%$ to their favorite option. Most people who split their 100 points chose adjacent options (see Table 1 for descriptives). Therefore, we scored the options from 1 (human alone) to 6 (algorithm alone) and multiplied them with the points given, resulting in a scale from 100 to 600 . Higher values represent a higher preference for algorithmic decision-making. A person giving 60 points to Option 3 (human decides, but always with input from the algorithm), and 40 points to Option 4 (algorithm decides, but always with input from the human), for example, has a score of $340\left(3{ }^{\star} 60+4\right.$ * $40=340$ ). A person favoring Option 3 with 60 points but leaning toward Option 2 (human decides, input from algorithm only in difficult cases) with 40 points has a score of 260 (2 $\star 40+3{ }^{*} 60=260$ ). This scale thus provides more fine-grained information than simply choosing one option.

\section{Results}

\section{Manipulation Checks}

As intended, the majority (91.7\%) perceived the ventilator scenario as the decision involving most morality. It was also perceived as the most severe scenario by $87.3 \%$. The other two scenarios did not differ as clearly from each other; the curfew scenario was perceived as the second-highest moral-laden (52\%) and least severe (61.5\%) scenario by most. The financial support scenario was perceived as involving the least morality $(55.1 \%)$ and as the second-most severe scenario (58.8\%). Since our hypothesis addressed the difference between the first and the other two scenarios, we considered the manipulation of the within-subjects variable as successful.

The manipulation check for perspective was answered correctly by $69.2 \%$ of the respondents for Scenario 1, by $64.9 \%$ for Scenario 2, and by $68 \%$ for Scenario 3. Respondents more often gave a wrong answer (between $20 \%$ and $33 \%$, depending on the scenario) than choosing the "don't know" option. Interestingly, respondents in the decision-maker condition recalled the condition they were in better than respondents in the target condition (75.3\% vs. $63.3 \%$ in Scenario 1, $74.6 \%$ vs. $55.3 \%$ in Scenario 2, $72.8 \%$ vs. $63.3 \%$ in Scenario 3 , all $\left.\chi^{2} s>22.13, p<.001\right)$. As preregistered, people who failed the manipulation check were excluded from the analysis; 1,127 participants were retained in the analysis (457 in the target condition, 670 in the decision-maker condition).

\section{Descriptive Results}

Before turning to the effects of the situational and individual variables, we briefly report the descriptive results. Table 1 shows that respondents overall leaned toward human decision-making. A closer look reveals, however, that algorithmic input is welcome; specifically, algorithmic input in all situations is favored over algorithmic input in difficult 
situations only. This suggests that participants want a human to make the final decision, but this human should team up with the algorithm.

\section{Effects of Scenario and Perspective}

A 2 (perspective: decision target versus decision-maker) $\times 3$ (Scenarios 1 to 3 ) repeated measures analysis of variance with repeated measures on the second factor and algorithm preference as dependent variable was conducted to test $\mathrm{H} 1$ and $\mathrm{H} 2$. In line with $\mathrm{H} 1$, there was a significant main effect of scenario, Huynh-Feldt corrected $F(1.994,2243.36)=40.48$, $p<.001, \eta_{\mathrm{p}}^{2}=.04$. Preference for algorithms was lowest in the ventilator scenario $(M=$ 286.46, $S E=3.75$, Bonferroni-corrected comparisons with both other scenarios $p<.001)$. The curfew scenario $(M=311.18, S E=4.13)$ and the financial support scenario $(M=$ $318.80, S E=3.84$ ) did not differ significantly, $p=.11$. In contrast to $\mathrm{H} 2$, the main effect of perspective was not significant, $F<1, p=.34$. There was, however, an interaction between perspective and scenario, Huynh-Feldt corrected $F(1.994,2243.36)=9.79, p<.001, \eta_{\mathrm{p}}{ }^{2}=$ .01. In the ventilator scenario, algorithm preference was higher in the decision-maker condition $(M=299.02, S E=4.77)$ than in the target condition $(M=273.90, S E=5.78, p=.001)$. In the financial support scenario, the means were almost identical $(M=318.79, S E=5.92$ in the target condition, $M=318.80, S E=4.89$ in the decision-maker condition, $p=.999$ ). In the curfew scenario, the mean in the target condition was somewhat higher $(M=314.34$, $S E=6.37)$ than in the decision-maker condition $(M=308.03, S E=5.26)$, but this difference was not significant, $p=.444$. H2 is thus partly supported, namely in the ventilator scenario.

\section{Influence of Individual Characteristics}

To examine the role of individual characteristics, we conducted a multilevel analysis, treating scenario as nested within participants. ${ }^{2}$ We included a random intercept for participants. In Step 1, we examined the effect of conventionalism (H3) and need for leadership (RQ) in addition to the effects of perspective, scenario, and the interaction effects. We controlled for being a member of a COVID-19 risk group and the risk to suffer from financial losses. In Step 2, we exploringly added the attitude subscales and knowledge about algorithms. Predictors were grand mean centered (see Enders \& Tofighi, 2007). Standardized regression coefficients are reported as effect size measures in Table 3 (see Lorah, 2018).

Table 3, Model 1, on the following page, shows the findings to be in line with H3: There was a negative relationship between conventionalism and algorithm preference. The answer to RQ1 is that need for leadership is positively related to a higher preference for algorithms.

\section{Exploratory Analyses}

In Model 2, we explored how the attitude toward algorithms affected algorithm preference. Several attitudes emerged as significant predictors: Algorithms were preferred more if they were evaluated as making more correct decisions, being fairer (in general and procedure-wise), and as integrating more aspects into their decision. Perceived knowledge about algorithms was significantly associated with preferring an algorithm, but effects were smaller than the effects of attitudes. Whereas the effects of scenario and the interaction with perspective still held, the effects of conventionalism and need for leadership were no longer significant when adding attitudes and knowledge to the model. 


\begin{tabular}{|c|c|c|c|c|c|}
\hline \multirow[b]{2}{*}{ Parameters } & \multicolumn{4}{|c|}{ Fixed effects } & \multirow{2}{*}{$\begin{array}{c}\text { Random } \\
\text { effects }\end{array}$} \\
\hline & Estimate & SE & $\mathbf{t}$ & $\boldsymbol{\beta}$ & \\
\hline \multicolumn{6}{|l|}{ Model 1} \\
\hline Intercept & 307.39 & 3.36 & 91.61 & .01 & 93.91 \\
\hline P: Decision-maker cond. & 7.48 & 6.87 & 1.09 & .03 & \\
\hline Sc: Ventilator cond. & -22.19 & 3.87 & $-5.74^{*}$ & -.08 & \\
\hline Sc: Financial support cond. & 8.62 & 3.87 & $2.23^{*}$ & .03 & \\
\hline Need for leadership & 10.12 & 2.81 & $3.61 *$ & .10 & \\
\hline Conventionalism & -7.21 & 2.34 & $-3.07^{*}$ & -.08 & \\
\hline Risk: health & -11.87 & 6.78 & -1.75 & -.05 & \\
\hline Risk: financial & -0.01 & 2.29 & -0.00 & -.00 & \\
\hline Perspective $\times$ Ventilator & 29.39 & 7.89 & $3.72^{*}$ & .05 & \\
\hline Perspective $\times$ Financial & 6.45 & 7.89 & 0.82 & .01 & \\
\hline \multicolumn{6}{|l|}{ Model 2} \\
\hline Intercept & 306.56 & 2.92 & 105.11 & .00 & 77.73 \\
\hline P: Decision-maker cond. & 8.94 & 5.97 & 1.50 & .03 & \\
\hline Sc: Ventilator cond. & -22.19 & 3.87 & $-5.74^{*}$ & -.08 & \\
\hline Sc: Financial support cond. & 8.62 & 3.87 & $2.23^{*}$ & .03 & \\
\hline Need for leadership & 2.92 & 2.48 & 1.17 & .02 & \\
\hline Conventionalism & -0.24 & 2.11 & -0.12 & -.00 & \\
\hline Risk: health & -5.65 & 5.92 & -0.95 & -.02 & \\
\hline Risk: financial & 2.67 & 2.00 & 1.33 & .03 & \\
\hline Perspective $\times$ Ventilator & 29.39 & 7.89 & $3.72^{*}$ & .05 & \\
\hline Perspective $\times$ Financial & 6.45 & 7.89 & 0.82 & .01 & \\
\hline A: correctness & 42.58 & 4.69 & $9.08^{*}$ & .24 & \\
\hline A: procedural fairness & 11.25 & 3.90 & $2.88^{*}$ & .07 & \\
\hline A: multifactorial DM & 10.37 & 3.07 & $3.38^{*}$ & .08 & \\
\hline A: manipulability & -3.44 & 3.20 & -1.08 & -.02 & \\
\hline A: general fairness & 23.31 & 3.97 & $5.87^{*}$ & .15 & \\
\hline Knowledge & 4.22 & 1.95 & $2.17^{*}$ & .05 & \\
\hline$x^{2}$ & 289.11 & & & & \\
\hline$p$ & $<.001$ & & & & \\
\hline $\mathrm{R}^{2}$ (marginal/conditional) & $.20 / .55$ & & & & \\
\hline
\end{tabular}

Note. 3,018 observations on 1,006 individuals. Higher values indicate a preference for an algorithm; $P=$ Perspective; Sc = Scenario; $\mathrm{A}=$ Attitude; DM = decision-making; Predictors are grand mean centered; All factors were coded using contrast coding (Gelman \& Hill, 2007). ${ }^{*}|t|>2.0$, indicating a significant effect. 


\section{Discussion}

The goal of this work was to examine the preference for different forms of human-algorithm teaming in algorithmic decision-making, exploiting the COVID-19 pandemic to create three salient and realistic scenarios. We examined the effects of situational (morality, perspective) and individual characteristics (conventionalism, need for leadership). In addition, the roles of attitudes toward algorithms and knowledge were explored. We found that the morality of a decision mattered: Participants showed the lowest preference for algorithmic decision-making in the ventilator scenario. Moreover, in this scenario decision targets were more reluctant to let algorithms decide than decision-makers. Higher conventionalism was associated with a lower preference for algorithmic decision-making, whereas a higher need for leadership was associated with a higher preference for algorithmic decision-making. Attitudes toward algorithms and knowledge contributed to predicting algorithm preference; thereby, decision quality and fairness were most important.

\section{Contributions to Prior Work}

Our results contribute to work on human-algorithm teaming by providing a measure that assesses the preference for certain combinations of human-algorithm teaming. Different patterns have been described before (Madni \& Madni, 2018; van der Waa et al., 2020), and it has been shown that people favor hybrid decision-making over pure algorithmic decision-making (Starke \& Lünich, 2020), but less was known about preferences for the different forms of human-algorithm teaming. We showed that participants overall preferred algorithmic advice in all decision situations to algorithmic advice only in difficult decision cases.

Prior work rarely looked at situational and individual characteristics that predict these preferences systematically. Another contribution of our work lies, thus, in varying the morality of the scenarios and the perspective of the participant. Work that merely looked at medical decision-making showed that algorithm preference is low in such scenarios (Bigman \& Gray, 2018; Longoni et al., 2019). Our results demonstrate that algorithm preference is lower in more moral-laden scenarios and that this effect is more pronounced when being the target of the decision. Decision-makers were less influenced by the morality of the scenario.

In contrast to our prediction, perspective mattered only in the most moral scenario. We did, thus, not find consistent evidence of an outcome favorability bias. Self-interest seems to bias decisions only in situations with high stakes. This seems to be in contrast with prior work, but in those studies, the outcomes were explicitly stated, and the dependent measure was perceived fairness, not the preference for algorithmic decision-making (Wang et al., 2020). Future research, however, is needed to explicitly test the role of self-interest and (expected) outcomes. The observation that perspective did not matter much also has implications for the interpretation of prior work. It suggests that the algorithm appreciation reported by Logg et al. (2019) may not be due to the decision-maker perspective but the moral-free judgment tasks. 
This study also contributes to work on individual predictors of algorithm preference; a topic that has been understudied compared to work on algorithm characteristics (Jussupow, 2020). We found the expected negative relationship between conventionalism and algorithm preference. With need for leadership, we found a positive association with algorithm preference: People with a higher need for leadership are not necessarily more interested in a strong human leader, but in clear and consistent guidance, which might be more easily achieved by an algorithm in times of crisis. During the COVID-19 pandemic, the German federal states employed different rules regarding school openings or curfews, and this diversity in rules was evaluated negatively by most of the population (COSMO, 2021).

Our exploratory analyses showed that attitudes toward algorithms mattered. Interestingly, the preference for algorithmic decision-making was not influenced by the perceived manipulability, although humans were perceived as easier to manipulate, which could be a reason for decision targets to prefer humans. Instead, quality of decision-making and perceived general fairness mattered most. Our participants considered algorithms as fairer than humans; nevertheless, one should be aware that algorithmic decisions mirror existing human biases such as prejudice or stereotypes and thus discriminate against certain groups (Noble, 2018; Zafar et al., 2019). The quality of algorithms depends on the training data used; if, for example, historic hiring data of a company that predominantly hired men are used to train an algorithm, the algorithm is likely to discriminate against women (O'Neil, 2016). Furthermore, deeper knowledge about algorithms was positively related to algorithm preference. Interestingly, knowledge was almost unrelated to attitudes but negatively associated with conventionalism. This shows again that conventionalism should be considered a potential barrier when trying to increase the public's algorithm acceptance.

After controlling for attitudes and knowledge, the effects of need for leadership and conventionalism were no longer significant, although the effects of scenario and perspective remained. The former is, however, not surprising because high attitude-behavior intention relationships are found when the correspondence between the attitude items and the behavior is high (Ajzen \& Fishbein, 1977). It is often assumed that attitudes are informed by knowledge; our data indicate that personality matters as well. Especially conventionalism showed negative associations with knowledge and attitudes. The results thus hint at a potential underlying process: People higher in conventionalism might show a lower algorithm preference because they are less willing to learn about algorithms (and other new things) and have more negative attitudes.

\section{Directions for Future Research}

Our experiment provides a starting point for future research. First, future research should look at underlying processes. When it comes to the decision targets, assumed outcome favorability and self-interest (versus interest in the fairest decision) should be assessed. In the decision-makers, looking at the mental models could be fruitful. Do decision-makers perceive the algorithm as an advanced tool, a teammate (Matthews et al., 2020), or do they even develop new mental models for human-algorithm teaming (Gambino et al., 2020)?

Second, work on human-algorithm teaming might focus more on the role of personality. The body of work has hitherto mainly looked at characteristics of the algorithmic teammate (e.g., agency, perceived autonomy, transparency), but the preference for a certain 
teaming-constellation might interact with individual characteristics. Considering both factors jointly would advance work on human-algorithm and more general human-machine teaming.

Third, future research could explore whether the results are specific to scenarios with a high salience and relevance like the COVID-19-related decisions we used during the pandemic.

Fourth, cross-cultural research could explore how far attitudes and the relationships between attitudes and knowledge are due to the specific media coverage of algorithmic decision-making in certain countries.

\section{Practical Implications}

The results have practical implications for policymaking. They show that the public is, in general, open to advice from algorithms, but that people prefer a human making the final decision. Since algorithms rarely make decisions completely on their own, governments planning to use algorithmic decision-making should use those only as advice-givers and communicate clearly that a human will make the final judgment.

Campaigns for increasing algorithm acceptance should especially target conventional people and consider that this group overall knows less about algorithms. For less moral decisions, it is important to know that decision-makers and targets did not differ in their preference of human-algorithm teaming and can thus be targeted with the same campaign. When it comes to moral decisions-as they occur frequently during a life-threatening pandemic-it is important to especially address the potential targets of the decisions because this group shows a lower preference for algorithmic input than decision-makers.

\section{Limitations and Strengths}

A limitation of the study is the relatively high number of failed manipulation checks. We, however, still had more than 1,100 people for analysis and thus almost twice the preregistered sample size of $n=602$ for $80 \%$ power. Another limitation is that we did not randomize the order of the scenarios. Some may consider it a limitation that the scenarios varied in the domain (e.g., health versus finance). However, this confound cannot be avoided because decisions threatening a person's life are inherently more moral than decisions involving finance because they imply more harm (Schein \& Gray, 2018). The same situation has been faced by other authors; Reniers et al. (2012), for example, used robbing a bank to pay the cancer medicine for one's wife versus eating chips while one should lose weight as a moral versus non-moral decision.

Furthermore, it is important to interpret our results considering the situation in Germany. Germany did a relatively good job in dealing with the pandemic at the time of data collection. It is thus unclear to which extent the results can be generalized to countries with an actual shortage of ventilators and a less tight-meshed social support system. The effects might be stronger in a country more strongly affected by COVID-19. Likewise, mean levels of need for leadership, conventionalism, and attitudes toward algorithms are likely to differ among countries; especially because issues such as racial discrimination by algorithms are less salient in Germany than, for example, the US (Noble, 2018). 
A strength of our study is the large and heterogeneous sample, covering a wide range of age, education levels, and employment status. Moreover, the decision-making scenarios were more realistic and salient than some of the advice-taking paradigms used in prior research on algorithm aversion.

To conclude, this study showed that the morality of a decision is a situational factor that determines the preference for different forms of algorithm-human teaming. The perspective decision target versus decision-maker mattered only in the most moral scenario. Moreover, personality factors such as need for leadership and conventionalism should be considered when studying attitudes toward algorithms and algorithm preference.

\section{Author Biographies}

Sonja Utz $(\mathrm{PhD})$ is the head of the social media lab at Leibniz-Institut für Wissensmedien in Tübingen and a full professor for communication via social media at University of Tübingen. Her research focuses on the effects of social and mobile media use, especially in knowledge-related contexts, as well as on human-machine interaction.

\section{https://orcid.org/0000-0002-7979-3554}

Lara N. Wolfers (MA in communication science, University of Hohenheim) is a $\mathrm{PhD}$ student working in the social media lab at the Leibniz-Institut für Wissensmedien in Tübingen, Germany. Her research focuses on the use of mobile and social media for coping with stress and media use in the family context.

(D) https://orcid.org/0000-0002-1074-1617

Anja S. Göritz (PhD) is full professor of psychology and leads the Department of Occupational and Consumer Psychology at Freiburg University in Germany. Her research focuses on web-based data collection and on technology-related changes of work. She holds and manages an online access panel (https://wisopanel.net), which also provided the participants of this study.

https://orcid.org/0000-0002-4638-0489

\section{Acknowledments}

Supported by the DFG Cluster of Excellence "Machine Learning-New Perspectives for Science," EXC 2064/1, project number 390727645

\section{Center for Open Science}

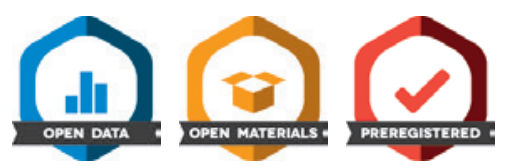

This article has earned the Center for Open Science badges for Open Data and Open Materials through Open Practices Disclosure. The authors have made their data and materials freely accessible at https://osf.io/cx6z8. The article also earned a Preregistered badge for having a preregistered design available at https://aspredicted.org/zv2m2.pdf. 


\section{References}

Ajzen, I., \& Fishbein, M. (1977). Attitude-behavior relations: A theoretical analysis and review of empirical research. Psychological Bulletin, 84(5), 888-918. https://doi. org/10.1037/0033-2909.84.5.888

Beierlein, C., Asbrock, F., Kauff, M., \& Schmidt, P. (2014). Die Kurzskala Autoritarismus (KSA-3). Ein ökonomisches Messinstrument zur Erfassung dreier Subdimensionen autoritärer Einstellungen. [The authorianism short-scale (KSA-3). An economic measurement instrument to capture three subdimensions of authoritarian attitudes]. GESIS-Working Papers, 35. Mannheim: GESIS.

Bigman, Y. E., \& Gray, K. (2018). People are averse to machines making moral decisions. Cognition, 181(12), 21-34. https://doi.org/10.1016/j.cognition.2018.08.003

Calhoun, C. S., Bobko, P., Gallimore, J. J., \& Lyons, J. B. (2019). Linking precursors of interpersonal trust to human-automation trust: An expanded typology and exploratory experiment. Journal of Trust Research, 9(1), 28-46. https://doi.org/10.1080/21515581.2 019.1579730

Castelo, N., Bos, M. W., \& Lehmann, D. R. (2019). Task-dependent algorithm aversion. Journal of Marketing Research, 56(5), 809-825. https://doi.org/10.1177/0022243719851788

COSMO. (2021, January). Zusammenfassung und Empfehlungen Welle 33. [Covid-19 Snapshot Monitoring: Summary and recommendations Wave 3]. https://web.archive. org/web/20210117120110/https://projekte.uni-erfurt.de/cosmo2020/web/summary/33/

COVID-19 pandemic. (2021, April 12). In Wikipedia. https://en.wikipedia.org/w/index. php?title=COVID-19_pandemic\&oldid=1017375094

Dietvorst, B. J., Simmons, J. P., \& Massey, C. (2015). Algorithm aversion: People erroneously avoid algorithms after seeing them err. Journal of Experimental Psychology: General, 144(1), 114-126. https://doi.org/10.1037/xge0000033

Dietvorst, B. J., Simmons, J. P., \& Massey, C. (2016). Overcoming algorithm aversion: People will use imperfect algorithms if they can (even slightly) modify them. Management Science, 64(3), 1155-1170. https://doi.org/10.1287/mnsc.2016.2643

Enders, C. K., \& Tofighi, D. (2007). Centering predictor variables in cross-sectional multilevel models: A new look at an old issue. Psychological Methods, 12(2), 121-138. https:// doi.org/10.1037/1082-989X.12.2.121

Erlanger, S. (2020, April 15). Coronavirus has lifted leaders everywhere. Don't expect that to last. The New York Times. https://web.archive.org/web/20200415211120/https://www. nytimes.com/2020/04/15/world/europe/coronavirus-presidents.html

Gambino, A., Fox, J., \& Ratan, R. A. (2020). Building a stronger CASA: Extending the computers are social actors paradigm. Human-Machine Communication, 1, 71-86. https:// doi.org/10.30658/hmc.1.5

Gelman, A., \& Hill, J. (2007). Data analysis using regression and multilevel/hierarchical models. Cambridge University Press.

Gollwitzer, M., Platzer, C., Zwarg, C., \& Göritz, A. S. (2020). Public acceptance of Covid19 lockdown scenarios. International Journal of Psychology. https://doi.org/10.1002/ ijop.12721

Göritz, A. S., Borchert, K., \& Hirth, M. (2021). Using attention testing to select crowdsourced workers and research participants. Social Science Computer Review, 39(1), 84-104. https://doi.org/10.1177/0894439319848726 
Grgic-Hlaca, N., Redmiles, E. M., Gummadi, K. P., \& Weller, A. (2018). Human perceptions of fairness in algorithmic decision making: A case study of criminal risk prediction. Proceedings of the 2018 World Wide Web Conference, 903-912. https://doi. org/10.1145/3178876.3186138

Grzymek, V., \& Puntschuh, M. (2019). Was Europa über Algorithmen weiß und denkt [What Europe knows and thinks about algorithms]. https://doi.org/10.11586/2019006

Jussupow, E., Benbasat, I., \& Heinzl, A. (2020). Why are we averse towards algorithms? A comprehensive literature review on algorithm aversion (No. 168). https://aisel.aisnet.org/ ecis2020_rp/168

Logg, J. M., Minson, J. A., \& Moore, D. A. (2019). Algorithm appreciation: People prefer algorithmic to human judgment. Organizational Behavior and Human Decision Processes, 151, 90-103. https://doi.org/10.1016/j.obhdp.2018.12.005

Longoni, C., Bonezzi, A., \& Morewedge, C. K. (2019). Resistance to medical artificial intelligence. Journal of Consumer Research, 46(4). https://doi.org/10.1093/jcr/ucz013

Lorah, J. (2018). Effect size measures for multilevel models: Definition, interpretation, and TIMSS example. Large-Scale Assessments in Education, 6, 8. https://doi.org/10.1186/ s40536-018-0061-2

Madni, A. M., \& Madni, C. C. (2018). Architectural framework for exploring adaptive human-machine teaming options in simulated dynamic environments. Systems, 6(4), 44. https://doi.org/10.3390/systems6040044

Mast, M. S. (2005). Interpersonal hierarchy expectation: Introduction of a new construct. Journal of Personality Assessment, 84(3), 287-295. https://doi.org/10.1207/ s15327752jpa8403_08

Matthews, G., Lin, J., Panganiban, A. R., \& Long, M. D. (2020). Individual differences in trust in autonomous robots: Implications for transparency. IEEE Transactions on Human-Machine Systems, 50(3), 234-244. https://doi.org/10.1109/THMS.2019.2947592

Messick, D. M., \& Sentis, K. (1983). Fairness, preference, and fairness biases. In D. M. Messick \& K. Cook (Eds.), Equity theory: Psychological and sociological perspectives (pp. 61-94). Praeger Publishers.

Mulder, M., \& Stemerding, A. (1963). Threat, attraction to group, and need for strong leadership: A laboratory experiment in a natural setting. Human Relations, 16(4), 317-334. https://doi.org/10.1177/001872676301600402

Newell, S., \& Marabelli, M. (2015). Strategic opportunities (and challenges) of algorithmic decision-making: A call for action on the long-term societal effects of 'datification.' The Journal of Strategic Information Systems, 24(1), 3-14. https://doi.org/10.1016/j. jsis.2015.02.001

Noble, S. U. (2018). Algorithms of oppression: How search engines reinforce racism. New York University Press.

O’Neil, C. (2016). Weapons of math destruction: How big data increases inequality and threatens democracy. Crown.

Reniers, R. L., Corcoran, R., Völlm, B. A., Mashru, A., Howard, R., \& Liddle, P. F. (2012). Moral decision-making, ToM, empathy and the default mode network. Biological Psychology, 90(3), 202-210. https://doi.org/10.1016/j.biopsycho.2012.03.009 
Schein, C., \& Gray, K. (2018). The theory of dyadic morality: Reinventing moral judgment by redefining harm. Personality and Social Psychology Review, 22(1), 32-70. https://doi. org $/ 10.1177 / 1088868317698288$

Smith, A. (2018). Public attitudes toward computer algorithms. https://web.archive.org/ web/20191030175600/https://www.pewresearch.org/internet/2018/11/16/publicattitudes-toward-computer-algorithms/

Starke, C., \& Lünich, M. (2020). Artificial intelligence for political decision-making in the European Union: Effects on citizens' perceptions of input, throughput, and output legitimacy. Data \& Policy, 2, e16. https://doi.org/10.1017/dap.2020.19

van der Waa, J., van Diggelen, J., Cavalcante Siebert, L., Neerincx, M., \& Jonker, C. (2020). Allocation of moral decision-making in human-agent teams: A pattern approach. In D. Harris \& W.-C. Li (Eds.), Engineering Psychology and Cognitive Ergonomics. Cognition and Design. HCII 2020. Lecture Notes in Computer Science, 12187, 203-220. Springer, Cham. https://doi.org/10.1007/978-3-030-49183-3_16

Wang, R., Harper, F. M., \& Zhu, H. (2020). Factors influencing perceived fairness in algorithmic decision-making: Algorithm outcomes, development procedures, and individual differences. Proceedings of the 2020 CHI Conference on Human Factors in Computing Systems, 1-14.

WHO. (2020). WHO announces COVID-19 outbreak a pandemic. Press release. https:// web.archive.org/web/20201223093341/https://www.euro.who.int/en/health-topics/ health-emergencies/coronavirus-covid-19/news/news/2020/3/who-announces-covid-19outbreak-a-pandemic

Zafar, M. B., Valera, I., Gomez-Rodriguez, M., \& Gummadi, K. P. (2019). Fairness constraints: A flexible approach for fair classification. Journal of Machine Learning Research, 20(75), 1-42.

\section{Footnotes}

1. We had two additional hypotheses and a research question on the role of individual outcome expectations. We realized, however, that we had not completely thought these through and not operationalized this variable in the best possible way. Based on reviewer feedback on a prior version on this manuscript, we decided to not include the weak theoretical justification and the results of these analyses. We adjusted the numbering of the hypotheses and research question. These analyses are, however, available on https://osf.io/cx6z8/. We also provide a table listing and justifying deviations from the preregistration on OSF.

2. In the preregistration, we had planned to do separate regression analyses per scenario. The main reason for this procedure was that the individual outcome expectations (see Footnote 1) were operationalized in different ways across the scenarios. Since we dropped this variable, we opted for the more appropriate multilevel approach that allows us to control for the nested data structure (scenarios nested within participants) and presents the results in a more compact way. The basic pattern is identical. 

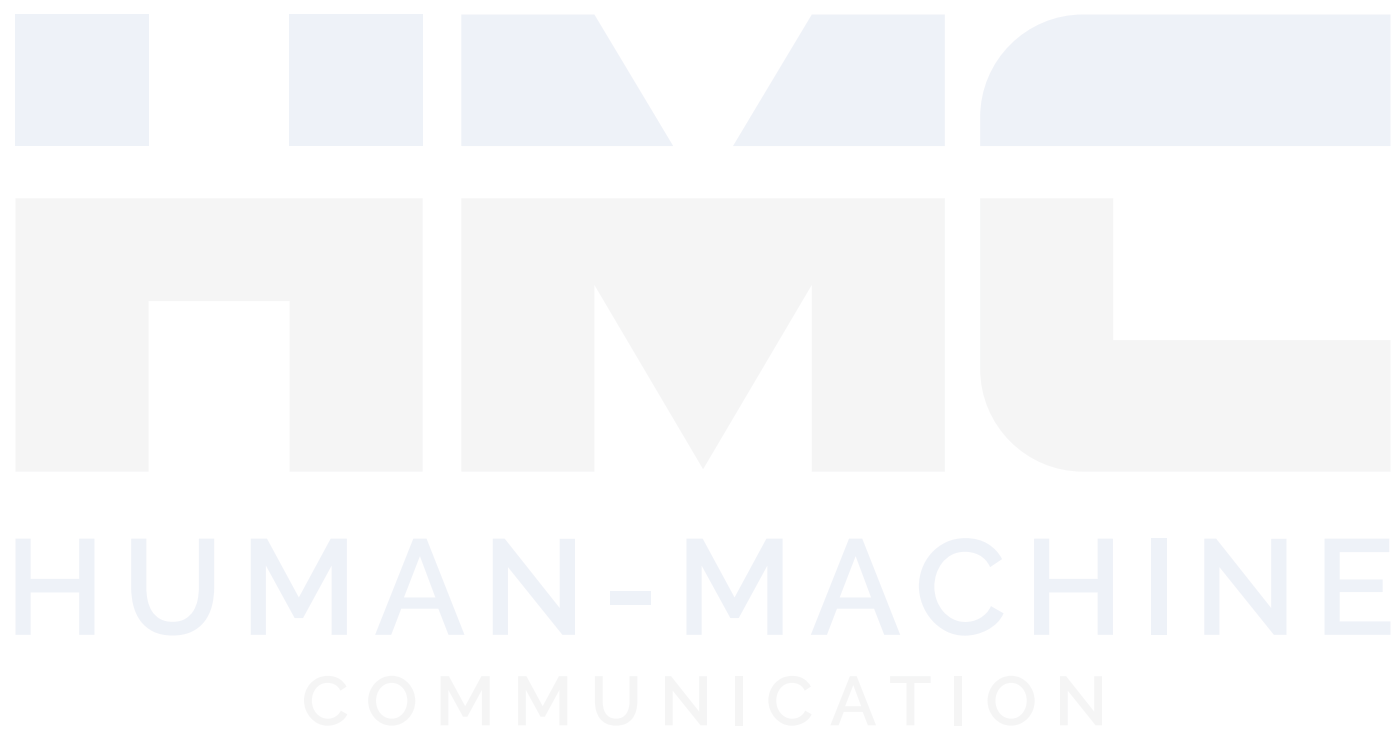